\title{
Laser and radio tracking for planetary science missions-a comparison
}

\author{
Dominic Dirkx ${ }^{1} \cdot$ Ivan Prochazka $^{2} \cdot$ Sven Bauer ${ }^{3} \cdot$ Pieter Visser $^{1} \cdot$ Ron Noomen $^{1} \cdot$ Leonid I. Gurvits $^{1,4}$. \\ Bert Vermeersen ${ }^{1}$
}

Received: 22 December 2017 / Accepted: 5 July 2018 / Published online: 24 July 2018

(c) The Author(s) 2018

\begin{abstract}
At present, tracking data for planetary missions largely consists of radio observables: range-rate (Doppler), range and angular position (VLBI/ $\triangle \mathrm{DOR}$ ). Future planetary missions may use Interplanetary Laser Ranging (ILR) as a tracking observable. Two-way ILR will provide range data that are about 2 orders of magnitude more accurate than radio-based range data. ILR does not produce Doppler data, however. In this article, we compare the relative strength of radio Doppler and laser range data for the retrieval of parameters of interest in planetary missions, to clarify and quantify the science case of ILR, with a focus on geodetic observables. We first provide an overview of the near-term attainable quality of ILR, in terms of both the realization of the observable and the models used to process the measurements. Subsequently, we analyse the sensitivity of radio Doppler and laser range measurements in representative mission scenarios for parameters of interest. We use both an analytical approximation and numerical analyses of the relative sensitivity of ILR and radio Doppler observables for more general cases. We show that mm-precise range normal points are feasible for ILR, but mm-level accuracy and stability in the full analysis chain are unlikely to be attained, due to a combination of instrumental and model errors. We find that ILR has the potential for superior performance in observing signatures in the data with a characteristic period of greater than 0.33-1.65 hours (assuming 2-10 mm uncertainty for range and $10 \mu \mathrm{m} / \mathrm{s}$ at $60 \mathrm{~s}$ for Doppler). This indicates that Doppler tracking will typically remain the method of choice for gravity field determination and spacecraft orbit determination in planetary missions. ILR data will be able to supplement the orbiter tracking data used for the estimation of parameters with a once-per-orbit signal. Laser ranging data, however, are shown to have a significant advantage for the retrieval of rotational and tidal characteristics from landers. Similarly, laser ranging data will be superior for the construction of planetary ephemerides and the improvement of solar system tests of gravitation, both for orbiter and for lander missions.
\end{abstract}

Keywords Interplanetary laser ranging $\cdot$ Radio tracking $\cdot$ Planetary missions

\section{Introduction}

For both Earth-orbiting and planetary missions, a variety of tracking observables is available from which the trajectory of the spacecraft can be reconstructed. A tracking data

$凶$ Dominic Dirkx

d.dirkx@tudelft.nl

1 Delft University of Technology, Kluyverweg 1, 2629 HS Delft, The Netherlands

2 Czech Technical University in Prague, Brehova 7, 11519 Prague 1, Czech Republic

3 Dep. 1 Geodesy and Remote Sensing, GeoForschungsZentrum Potsdam (GFZ), Telegrafenberg, Potsdam, Germany

4 Joint Institute for VLBI ERIC, PO Box 2, 7990 AA Dwingeloo, The Netherlands type that will become available for future planetary missions is Interplanetary Laser Ranging (ILR) (Degnan 2002). The technology for such a system derives strongly from Satellite Laser Ranging (SLR; Pearlman et al. 2002), Lunar Laser Ranging (LLR; Murphy 2013) as well as Laser Time Transfer (LTT; Prochazka et al. 2011; Exertier et al. 2014). The key difference between SLR/LLR and ILR is that ILR requires a transponder on both the ground and space segments, as opposed to the purely passive space segment in SLR/LLR (retroreflectors).

Currently, deep-space missions largely rely on the use of radio tracking for their orbit determination and the associated parameter estimation. In particular, Doppler data have been the primary data type for this application (e.g. Konopliv et al. 2011; Iess et al. 2012; Mazarico et al. 2014). Radiobased range data supplement the Doppler data by providing 
the absolute distance between spacecraft and ground station. The angular position of the spacecraft in the sky can be measured by means of Very Long Baseline Interferometry (VLBI) (Duev et al. 2012). In the so-called PRIDE (Planetary Radio Interferometry and Doppler Experiments) setup, VLBI and Doppler data are obtained concurrently (Duev et al. 2016). Unlike the Doppler data, range and VLBI data are used primarily for the estimation of solar system ephemerides (Fienga et al. 2009; Jones et al. 2015; Dirkx et al. 2017), which provide crucial input for experimental relativity (e.g. Will 2014). The selection of ILR as a tracking type in future missions is contingent upon its data being able to provide scientific results that are complementary/supplementary to, or competitive with, the results obtained from existing systems, such as those mentioned above.

There have been a number of experimental demonstrations of ILR, both in one-way (Abshire et al. 2006; Neumann et al. 2008; Noda et al. 2017) and in two-way (Smith et al. 2006) modes. The only operational implementation of ILR to date has been on the Lunar Reconnaissance Orbiter (LRO), using the laser altimeter system (Zuber et al. 2010; Bauer et al. 2016; Mao et al. 2017). None of the demonstrations of ILR have been performed with dedicated hardware, and the attainable measurement accuracy has not yet been pushed to the limit of state of the art. In recent years, there has been substantial development in LTT (Exertier et al. 2014; Samain et al. 2015; Exertier et al. 2017; Prochazka et al. 2017a), which is in many ways similar to a transponder ranging system. Laser communication technology is also maturing for use in planetary missions, as demonstrated by the LLCD demonstrator on the LADEE lunar orbiter. As an ad hoc product, LLCD communications data were used to obtain two-way ranging data with a precision of several $\mathrm{cm}$ (Stevens et al. 2016).

Thus far, analyses of various aspects of ILR have focussed on single- mission concepts (Turyshev et al. 2004; Chandler et al. 2005; Merkowitz et al. 2007; Luo et al. 2009; Christophe et al. 2009; Turyshev et al. 2010; Birnbaum et al. 2010; Oberst et al. 2012; Iorio 2013; Dirkx et al. 2014b), or operational and data analysis aspects (Folkner and Finger 1990; Degnan 1996, 2002, 2008; Schreiber et al. 2009; Dirkx et al. 2014a, 2015, 2016b; Bauer et al. 2016, 2017; Mao et al. 2017). Additionally, the majority of these analyses have focused on using the laser ranging data to improve solar system tests of relativity. Considering the great wealth of information on, for example, planetary geodesy that radio tracking has provided, it is natural to extend the analysis of ILR to planetary science objectives.

Our goal is to provide a comparison of radio Doppler and laser range data for planetary missions and to identify the areas where the addition of ILR data would be beneficial for attaining the scientific objectives of planetary missions. A preliminary set of results is given by Dirkx (2015). Instead of focusing on a single-mission concept in detail, we take a broad view and quantitatively analyse the relative strength of the range and Doppler observables for parameters of interest in a variety of planetary missions. As a result, we identify classes of science products/mission scenarios for which ILR will be a competitive design option.

We give an overview of tracking data in Sect. 2 and discuss the expected error budget of ILR in Sect. 3. In Sect. 4, we present the methods we use to compare ILR and Doppler data. We use both an analytical approach and a numerical covariance analysis based on simulated orbit determination/parameter estimation. The numerical technique serves to identify the level of applicability of the analytical approach and to provide guidelines on how to apply it in mission design and analysis. In Sect. 5, we show the results of our comparison of the two data types. In Sect. 6, we discuss the implications of these results for the use of ILR data, with a focus on geodetic observables. We conclude with a discussion on the overall science case of ILR in Sect. 7.

\section{Planetary tracking data}

In this section, we give a general overview of planetary tracking data, including the models for both range and Doppler observables.

\subsection{Radio data}

The typical precisions of radio tracking data are currently at the level of $0.02-0.1 \mathrm{~mm} / \mathrm{s}$ at 60 -s integration time for range-rate measurements at X-band (e.g. Thornton and Border 2000; Marty et al. 2009; Konopliv et al. 2011; Iess et al. 2014; Bocanegra-Bahamón et al. 2018) and 0.5-5 m for range measurements (e.g. Thornton and Border 2000; Folkner et al. 2014). Detailed discussions on sources of both systematic errors and random noise are given by Thornton and Border (2000); Moyer (2000); Asmar et al. (2005); Iess et al. (2014); Molera Calvés et al. (2014). For Doppler measurements, the systematic errors are typically close to negligible (e.g. Iess et al. 2014). In contrast, the level of systematic errors of radio range measurements can be quite large, comparable to the random noise, at the $1 \mathrm{~m}$ level.

The radio tracking noise is dominated by the propagation effects in the interplanetary medium and depend strongly on the solar elongation angle. For Mars Express, Duev et al. (2016) show that PRIDE Doppler noise at X-band is indeed dominated by these effects, with a median one-way value of $\sim 30 \mu / \mathrm{s}$ at 10 -s integration time. Combining observations at multiple wavelengths allows the removal of the majority of these errors (Reasenberg et al. 1979; Bertotti et al. 2003). The combined $X$ - and Ka-band approach was/is used for Cassini (Kliore et al. 2004) and Juno (Iess et al. 2018), and is anticipated for use on the BepiColombo and JUICE mis- 
sions, which have tracking data quality requirements of 0.01 $\mathrm{mm} / \mathrm{s}$ at 60 -s integration time and $3 \mu \mathrm{m} / \mathrm{s}$ at 1000 -s integration time. Additionally, these missions will use an advanced radio ranging system, allowing two-way range measurements with a predicted accuracy of down to $20 \mathrm{~cm}$.

\subsection{Laser ranging-measurement concepts}

SLR has been used for Earth-orbiting satellites for more than 50 years (Pearlman et al. 2002) and provides two-way range data with sub-cm accuracy. In ILR, the use of retroreflectors is no longer feasible due to the large target distance requiring an active system in both the ground and space segments.

Two main types of active laser ranging systems (sometimes termed transponder laser ranging) can be distinguished for use in planetary missions (Degnan 2002; Birnbaum et al. 2010, see Fig. 1):

- One-way laser ranging. A laser pulse is transmitted from a ground station and detected by a (satellite-based) receiver (or the other way around). An important issue with this method is that the transmission and reception times are measured by different, unsynchronized, clocks (Bauer 2017).

- Two-way asynchronous laser ranging. Both the space and ground segment fire laser pulses towards one another independently. By pairing a range measurement from the up- and down-link, a two-way range measurement is obtained (Birnbaum et al. 2010), which does not suffer from the clock error issue of a one-way system.

Two-way asynchronous laser ranging has been the method of choice in most ILR mission proposals, due to its higher data quality and less stringent clock requirements. In this article, our main focus is on two-way systems.

Both the one- and two-way observables are created from time tags of the transmission $\left(\tau_{t}\right)$ and reception $\left(\tau_{r}\right)$, in their local proper time scales. ${ }^{1}$ The error-free two-way raw range measurement is then created from the coordinate times $t$ as:

$s\left(t_{t}\right)=c\left(t_{r}-t_{t}\right)$

Due to uncalibrated errors in the measurements (Sect. 3) of $\tau_{t}$ and $\tau_{r}$, the range measurement quality is degraded. We

\footnotetext{
${ }^{1}$ In the context of this article, we use the symbol $t$ to denote either a coordinate time such as Barycentric Coordinate Time (TCB) or a scaled coordinate time such as Dynamical Barycentric Time (TDB). Although TDB is decidedly not a coordinate time, as discussed in detail by Klioner (2008), the distinction is not relevant for the purpose of our discussion. Details of the conversion between coordinate and proper time scales are discussed by Soffel et al. (2003), while the influence on the data analysis is discussed by Dirkx et al. (2016b).
}

denote the measured time (transformed to coordinate time) as $\tilde{t}$, so that the measured range $\tilde{s}$ becomes:

$$
\begin{aligned}
\tilde{s}\left(\tilde{t}_{t}\right) & =c\left(\tilde{t}_{r}-\tilde{t}_{t}\right) \\
& =s\left(t_{t}\right)+\varepsilon_{s}
\end{aligned}
$$

where $\varepsilon_{s}$ is the lumped range error. Note that this range value includes the effects of atmospheric and relativistic effects.

\subsection{Observation models}

We will denote the one-way range observation between point $A$ (transmitter) and point $B$ (receiver) by $s_{B A}^{(1)}$. From their position functions, denoted $\mathbf{r}_{A}(t)$ and $\mathbf{r}_{B}(t)$, respectively, the one-way range is obtained from:

$s_{B A}^{(1)}\left(t_{r}=t_{2}\right)=\left|\mathbf{r}_{B}\left(t_{2}\right)-\mathbf{r}_{A}\left(t_{2}-\frac{s_{B A}^{(1)}}{c}\right)\right|+\Delta s_{B A}^{(1)}\left(t_{1}, t_{2}\right)$

where the formulation is referenced to the reception time $t_{r}$, here equal to a given $t_{2}$. The term $\Delta s_{B A}^{(1)}$ denotes range corrections due to, for example, propagation medium and relativistic effects.

For a one-way range-rate (or Doppler) observable, denoted here as $\dot{s}_{B A}^{(1)}$, with an integration time denoted by $\Delta t_{i}$, the observable is modelled as (Moyer 2000):

$\dot{s}_{B A}^{(1)}\left(t_{r}=t_{4}\right)=\frac{s_{B A}^{(1)}\left(t_{r}=t_{4}\right)-s_{B A}^{(1)}\left(t_{r}=t_{2}\right)}{\Delta t_{i}}$

where $\Delta t_{i}=t_{4}-t_{2}$. Here, two one-way range observables with reception times $t_{4}$ and $t_{2}$ (and associated transmission times $t_{3}$ and $t_{1}$ ) are used. Typical values of $\Delta t$ are $1-60 \mathrm{~s}$, but may be $>1000 \mathrm{~s}$ in certain cases.

The two-way range is modelled as the combination of two one-way ranges:

$s_{B A}^{(2)}\left(t_{r}=t_{4}\right)=s_{A B}^{(1)}\left(t_{r}=t_{4}\right)+s_{B A}^{(1)}\left(t_{r}=t_{2}\right)+c \delta t_{B}$

$t_{2}=t_{4}-\frac{s_{A B}^{(1)}\left(t_{r}=t_{4}\right)}{c}-\delta t_{B}$

where $\delta t_{B}$ represents the delay between the reception and retransmission of the signal at station $B$, typically $<1 \mathrm{~ms}$ for radio data (Bertone et al. 2018), up to the order of 1 minute for ILR (Dirkx et al. 2015).

\section{Laser ranging data-error sources}

The error budgets of radio tracking systems are well understood and quantified (Sect. 2.1). Here we analyse and discuss the various sources of error in ILR measurements/analysis. 
Fig. 1 Schematic representation of laser ranging concepts. Thick lines indicate signals to trigger the transmission of a laser pulse. a One-way laser ranging, b two-way asynchronous laser ranging. Figure adapted from Dirkx (2015)

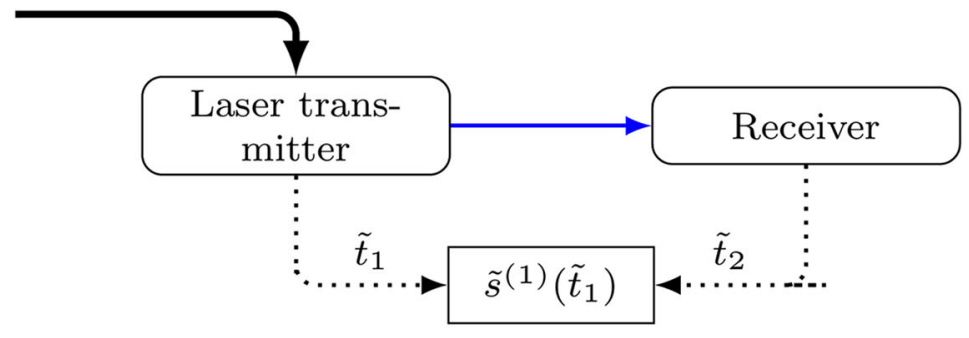

(a)

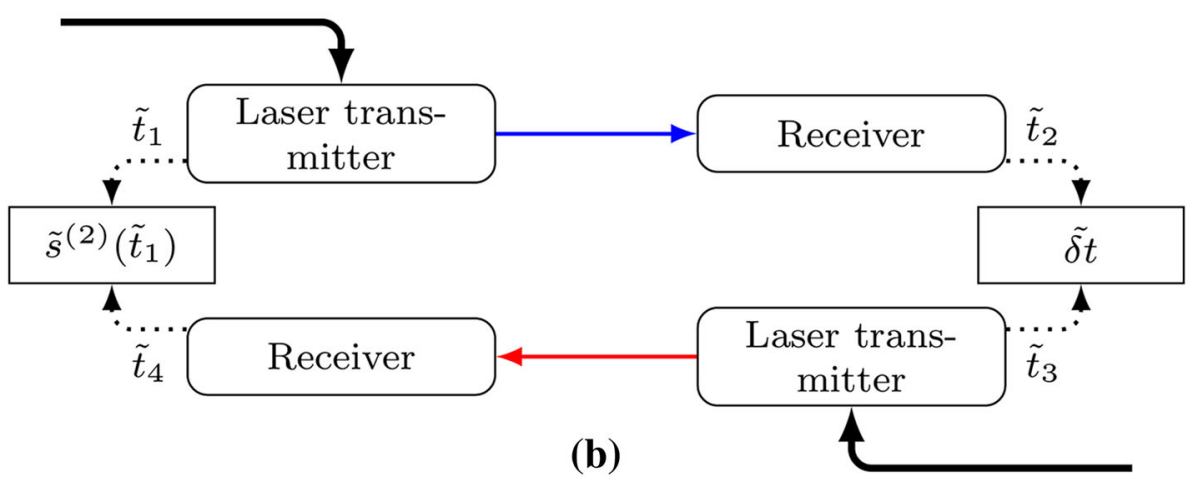

(b)

for SLR, as the incoming pulse energy temporal profile is more predictable in ILR.

The primary measurement uncertainty of ILR is a convolution of two main contributors: the laser pulse profile and the detector impulse response. For a pulse with a perfectly Gaussian temporal profile and single-photon intensity detection energies, the pulse profile introduces a purely Gaussian measurement error (Murphy 2001; Dirkx et al. 2014a). The one-way range precision limit due to only this effect is 1.3$13 \mathrm{~mm}$ single-shot root mean square (RMS) for laser pulses with a full-width half maximum (FWHM) of 10-100 ps, respectively. Note that the actual single-shot precision may be limited by the detector random error (Sect. 3.2).

In cases where the number of detectable photons is larger than one, additional range biases at the several $\mathrm{mm}$ level may be incurred (Dirkx et al. 2014a), if traditional threshold detection is used. Degnan (2017) has proposed the use of centroid detection that could be used to overcome this bias, by combining all incoming detections from a single pulse into a single waveform. In ILR, however, detection energies are expected to be at the single-photon level.

The contribution due to the atmospheric turbulence (Gardner 1976) is in most cases below the $0.5 \mathrm{~mm}$ level (Kral et al. 2005) (see Sect. 3.3 for discussion of troposphere model errors).

In SLR/LLR, the retroreflector signature causes significant distortion of the temporal pulse shape (Otsubo and Appleby 2003), which is not the case for ILR. Therefore, this aspect of the data stability could be better for ILR than

\subsection{Measurement errors}

For ILR, the hardware-derived error sources are similar to those of SLR, which were summarized by Exertier et al. to that given there, we note several recent changes in the coming sections. An important difference between SLR and ILR stems from the fact that in ILR part of the active hardware is on the space segment, and no passive reflectors are used.

For the characterization of the space segment, we rely in part on the development of space-grade detection systems that are currently in operation, such as T2L2 (Exertier et al. 2014), and those that are under development, such as the single-photon ELT (Prochazka et al. 2012). Although the optical components of T2L2 and ELT are very different from those in an ILR system, the stable single-photon detection system has very similar characteristics.

The measurement error due to the detector, often a silicon photon avalanche diode (SPAD), is at $3-6 \mathrm{~mm}$, the largest contributor to hardware-induced error budget of SLR (Exertier et al. 2006). Space-grade detectors showing sub-ps stability have been developed for the ELT project (Prochazka et al. 2011; Kodet and Prochazka 2012). The contribution to the ILR precision of the best photon-counting detectors is typically 3 mm RMS (Prochazka et al. 2017b). (2006). Although much of the error budget remains close 
Exertier et al. (2006) give values of several $\mathrm{mm}$ for the influence of jitter in the event timer. A novel type of event timer, developed and applied by Panek et al. (2010), Prochazka et al. (2011), provides sub-ps precision and a stability of several fs over a period of minutes to hours. The use of this technology allows the event timer to have an almost negligible contribution to the range error budget.

For ILR, the influence of clock noise is substantially different from SLR/LLR (Degnan 2002; Dirkx et al. 2015). For two-way systems, the clocks only need to be sufficiently stable over short periods of time (two-way light time). A stability of about $10^{-15}$ over a typical ILR light time of $1000 \mathrm{~s}$ will result in 1-ps timing error (0.3-mm one-way range error) and is achievable by H-masers (e.g. Dehant et al. 2017). For the space segment, stability is only required over the time $\delta t$, putting sub-mm errors well within the capabilities of present spaceborne systems. A one-way range system requires clocks at both ends of the link to be stable over longer time periods (Bauer et al. 2016), making clock noise a significant issue.

Delays in various components of the electrical and optical system of the detection assembly must be accurately characterized to realize a high-quality range measurement. Kirchner and Koidl (2014) show that ground station calibration consistency on short time scales is at the several ps level averaged over $10 \mathrm{~s}$, comparable to the value of $3 \mathrm{ps}$ given by Prochazka et al. (2012) for ELT. Both are in line with the value of $1 \mathrm{~mm}$ given by Exertier et al. (2006). Nevertheless, consistently obtaining mm-level system calibration on the space segment will be challenging.

Data from existing two-way ILR experiments cannot be used to set up a measurement error budget. The only two-way ILR experiment thus far used the non-dedicated hardware on the MESSENGER spacecraft (Smith et al. 2006), which is not representative of the state of the art. Two-way links have been demonstrated on laboratory scales. Chen et al. (2013) obtain range measurement errors below the $0.2 \mathrm{~mm}$ level (averaged over 1000 measurements). Blazej et al. (2014) have shown time transfer with an accuracy of $3 \mathrm{ps}(\approx 1 \mathrm{~mm})$ using two representative ground segment hardware packages. These experiments show the capabilities of laboratory-scale experiments with well-controlled hardware, indicating the potential for (sub-) $\mathrm{mm}$ range accuracy.

\subsection{Data analysis uncertainties}

Even in the case of perfect range measurements $\left(\varepsilon_{s}=0\right)$, the science products obtained from the data will not be error-free. Errors in the evaluation of Eq. (4) will degrade the fidelity of the results.

The position of the ground station in the Geocentric Celestial Reference System (GCRS) requires a time-dependent position in the International Terrestrial Reference Frame (ITRF), as well as a rotation between the two. Inaccuracies in these models limit the accuracy of the ground station position function at the sub-cm level (Altamimi et al. 2011; Rothacher et al. 2011; Sośnica et al. 2013).

ILR analysis must be performed in the Barycentric Celestial Reference System (BCRS). As a result, uncertainties in the a priori Earth ephemeris will enter the error budget of the ground station position model. The ephemeris of the Earth is orders of magnitude less accurate than the expected $\mathrm{cm}$ accuracy of ILR measurements (Fienga et al. 2011). This indicates that the Earth's ephemeris should be among the estimated parameters during ILR data analysis.

For landers on solar system bodies, the general issues in modelling the barycentric state are of a similar nature as for Earth ground stations. Depending on the target body, however, the uncertainty may be limited and accounted for by the addition of a number of estimated parameters. In fact, the signatures of these effects will often be key science objectives of the lander tracking (e.g. Le Maistre et al. 2013; Dirkx et al. 2014b). Fulfilling the modelling requirements may require significant theoretical work. For planetary dynamics, the uncertainty in asteroid masses and orbits is presently the limiting factor in the dynamical models (Fienga et al. 2009). For the dynamics of natural satellite systems, the consistent coupling between translational and rotational dynamics and tidal deformation will be challenging to model at the $\mathrm{mm}$ level (e.g. Dirkx et al. 2016a).

For orbiters, uncertainties in non-conservative forces, as well as target body gravity field variations (e.g. Marty et al. 2009), can limit the accuracy to which the dynamics can be modelled. This requires the state estimation to be performed arc-wise (A typical arc length is several days). Dynamical model error can become the dominant source of uncertainty in the estimated parameters and is a key reason for the true estimation errors often being significantly higher than the formal estimation errors (e.g. Konopliv et al. 2011; Mazarico et al. 2014).

Finally, models for tropospheric correction (as part of $\Delta s_{B A}$ ) have an accuracy of 5-8 $\mathrm{mm}$ (Exertier et al. 2006). This level can be reduced to the (several) $\mathrm{mm}$ level using ray-tracing models (Hulley and Pavlis 2007). Detailed models for relativistic range corrections have been developed (e.g. Teyssandier and Poncin-Lafitte 2008), so model uncertainties for this contribution of $\Delta s_{B A}$ will be negligible if state-ofthe-art models are applied.

\subsection{Total uncertainty—summary}

In Sects. 3.1-3.3, we have presented the main error sources that enter the data realization and analysis chain of ILR. The main sources of measurement error are the detector uncertainty (at $3 \mathrm{~mm}$ ), and the finite pulse length (3-13 mm RMS, for laser pulses 10-100 ps FWHM, respectively). 
As a result, 1.0-4.3-mm precision averaged over 10 measurements (for 10-100 ps pulse length) may be achieved. Considering the existing detector and timing devices performance (Sect. 3.2), a limiting precision (but not accuracy) $<0.1 \mathrm{~mm}$ can be achieved when averaging higher number of individual single-photon measurements.

Hardware imperfections, as they are deduced from current SLR and space-based laser transmitter and detector systems, will induce systematic errors at the several $\mathrm{mm}$ level, as is the case for SLR.

It is especially the instabilities in the systems that will be an issue for the quality of ILR data (as well as for SLR/LLR). A system bias that is constant for an extended time interval can be mitigated by the estimation of a single long-arc range bias. Instabilities (e.g. random walk behaviour) cannot be removed in this manner without introducing an excessive number of parameters. Therefore, ILR data accuracy will be limited to the level of several $\mathrm{mm}$ at best. Considering the current performance of SLR systems, sub-cm accuracy will be feasible. A major design driver for the space segment will be the stable system delay calibration in an (inter)planetary environment. Existing model errors in tropospheric correction and ground station positioning will limit ILR data modelling to the several $\mathrm{mm}$ level (Sect. 3.3). These issues are also crucial in space geodesy and are a topic of active research.

Dynamical model errors of both the space segment and the Earth will limit the accuracy to which the data can be interpreted. The degree to which these errors will affect the estimation of parameters of interest is strongly dependent on the correlation of the signatures of these parameters with the model errors. This error source is similar for both Doppler and range data and can prevent a data set from being exploited to its full potential.

\section{Data-type comparison methodology}

In this section, we present the methods we use to compare ILR with radio data. We outline our concept for analytical comparison and numerical covariance analysis in Sects. 4.1 (based on Dirkx 2015) and 4.2, respectively.

\subsection{Analytical approach}

The sensitivity of an observable $h$ to a parameter $q$ is quantified by its associated partial derivative $\partial h / \partial q$ (e.g. Montenbruck and Gill 2000). Since the range observable $s$ and range-rate observable $\dot{s}$ are related through Eq. (5), we have:

$\frac{\partial \dot{s}(t)}{\partial q}=\frac{1}{\Delta t_{i}}\left(\frac{\partial s\left(t+\Delta t_{i}\right)}{\partial q}-\frac{\partial s(t)}{\partial q}\right)$
To quantitatively compare the data types, we define a signal-to-noise (SNR) criterion for an observable $h$ and a parameter $q$, denoted $\mathrm{SNR}_{h ; q}$, which is computed as follows:

$\mathrm{SNR}_{h ; q}=\left|\frac{1}{\sigma_{h}} \frac{\partial h}{\partial q}\right|$

where $\sigma_{h}$ is the noise level of the measurement $h$.

Now, we define the following figure of merit to compare the relative sensitivity of range and Doppler observables to a parameter $q$ :

$\Xi_{q}=\frac{\max _{t}\left(\mathrm{SNR}_{\dot{s} ; q}\right)}{\max _{t}\left(\mathrm{SNR}_{s ; q}\right)}$

where the maximum is taken over the observational period. To first order, we can set $\Xi_{q}<1$ as a criterion when ILR would become a feasible alternative to Doppler data for determining a parameter $q$. The interpretation of numerical values of this criterion is discussed in Sect. 6.1.

We start by using an analytical model for Eq. (8), in which the influence of a parameter $q$ is manifested in the range measurements as a purely sinusoidal signal of amplitude $A$ and angular frequency $\omega$ (period denoted as $T$ ), so that:

$\frac{\partial s}{\partial q}=A \sin (\omega t)$

and we obtain the following from Eq. (8):

$\frac{\partial \dot{s}(t)}{\partial q}=\frac{A}{\Delta t_{i}}\left(\left(\cos \left(\omega \Delta t_{i}\right)-1\right) \sin (\omega t)+\sin \left(\omega \Delta t_{i}\right) \cos (\omega t)\right)$.

This approximates the situation where all planets are in circular orbits around a static Sun, with the spacecraft in a circular orbit around one of these bodies, and the parameter $q$ imparting an $N$-cycles-per-orbit sinusoidal signal on the data (with $N$ an integer). In the data, the orbital frequencies of the spacecraft, Earth and the target planet will then all be visible. ${ }^{2}$ These assumptions are a reasonable approximation for our analysis, as validated in Sect. 5.2 from numerical results. In "Appendix A", we discuss how to extend the method to elliptical orbits.

For $\Delta t \ll T$, we obtain the following from Eq. (12):

$\lim _{\omega \Delta t_{i} \rightarrow 0}\left(\max _{t}\left(\frac{\partial \dot{s}}{\partial q}\right)\right)=\frac{2 \pi A}{T}=\omega A$

\footnotetext{
${ }^{2}$ For various parameters, the sinusoidal signature will be modulated by a linear trend, increasing the amplitude with time. However, if the observation time is much larger than the period of the signal $T$, the impact of this linear trend on Eq. (10) will be small.
} 
so that:

$\left.\Xi_{q}\right|_{\Delta t \ll T} \approx \frac{\sigma_{s}}{\sigma_{\dot{s}}} \omega$

We apply this limit approximation in Sects. 5 and 6 to compare the analytical and numerical approaches.

\subsection{Covariance analysis-numerical models}

To assess the validity of our analytical results and gain insight into how the results obtained from them should be interpreted, we perform covariance analyses (e.g. Montenbruck and Gill 2000; Milani and Gronchi 2010) for a number of representative cases. We generate formal errors for a set of parameters when using only Doppler data and when using only range data. We denote the formal error of parameter $p$, using data type $h$, as $\epsilon_{p, h}$. $^{3}$

We simulate two scenarios: a Mars lander mission and a Mars dual-orbiter mission. The orbits of the spacecraft are both low-altitude, nearly polar and nearly circular, with initial condition $e=0.01$ for both; $a=3850 \mathrm{~km}$ and $i=88^{\circ}$ for one orbiter and $i=92^{\circ}$ and $a=3800 \mathrm{~km}$ for the other orbiter (similar to spacecraft such as Mars Odyssey and Mars Reconnaissance Orbiter). The lander is placed equatorially. For both cases, we assume a simplified Mars rotation model, with fixed pole right ascension and declination $\alpha_{M}$ and $\delta_{M}$, and a fixed rotation rate $\omega_{M}$.

For the orbiter simulation, we estimate the initial states $\mathbf{x}_{i}$ of both orbiters $(i=1,2)$ w.r.t. Mars' centre of mass over 1-week arcs, with each arc $j$ starting at time $t_{j}$ over a 2year period ( $j=1 . .104)$. For both the lander and the orbiter simulations, we estimate Mars' dynamics over a single 2-year arc w.r.t. the barycentre $\mathbf{x}_{M}\left(t_{0}\right)$ (see Table 1).

We set up our state transition matrix $\Phi\left(t, t_{0}\right)=\partial \mathbf{x} / \partial \mathbf{x}\left(t_{0}\right)$ (with $\left.\mathbf{x}=\left[\mathbf{x}_{M} ; \mathbf{x}_{1}\left(t_{1}\right) ; \ldots ; \mathbf{x}_{1}\left(t_{104}\right) ; \mathbf{x}_{2}\left(t_{1}\right) ; \ldots ; \mathbf{x}_{2}\left(t_{104}\right)\right]\right)$ in such a way that the coupling terms $\partial \mathbf{x}_{i}(t) / \partial \mathbf{x}_{M}\left(t_{0}\right)$ are referenced to the correct arc, so that for a given arc $j$ :

$\frac{\partial \mathbf{x}_{i}(t)}{\partial \mathbf{x}_{M}\left(t_{0}\right)}=\delta_{i j} \frac{\partial \mathbf{x}_{i}(t)}{\partial \mathbf{x}_{i}\left(t_{j}\right)} \frac{\partial \mathbf{x}_{i}\left(t_{j}\right)}{\partial \mathbf{x}_{M}\left(t_{0}\right)}, t \in\left[t_{j}, t_{j+1}\right)$

with $\delta_{i j}$ the Kronecker delta function. By doing so, the estimation of spacecraft state and natural body state can be done concurrently, as the influence of a change of natural body state is directly mapped to a change in (barycentric) spacecraft state.

This approach is in contrast to the typical approach of orbit determination and ephemeris generation, where the spacecraft's orbit is first estimated using Doppler data only, and the

\footnotetext{
${ }^{3}$ Note that we use the symbols $\varepsilon$ (lumped range error) and $\epsilon$ to represent different physical quantities.
}

planetary ephemerides are then estimated using range/VLBI data (without adjusting the spacecraft orbit). In this traditional approach, the direct coupling between the planetary and spacecraft orbits is omitted. When incorporating ILR, however, the laser data will have significant and useful information on the dynamics of both natural and artificial bodies, requiring the coupling to be incorporated into the simulations. Except for the modification in the computation of $\Phi\left(t, t_{0}\right)$ as in Eq. (15), our covariance analysis follows that outlined by, for example, Montenbruck and Gill (2000).

The list of parameters we estimate for both scenarios is given in Table 1. The relevance of the parameters in the context of Mars missions is discussed by, for example, Konopliv et al. (2011), Rivoldini et al. (2011). The notation $(C, S)_{l, m}$ is used as a shorthand for the combination of spherical harmonic coefficients $C_{l m}$ and $S_{l m}$ of Mars. For the Sun, we consider only the degree-two zonal coefficient $J_{2, \odot}$. The Love numbers are denoted as $k_{l m}$, and $\beta$ denotes the PPN parameter (Will 2014). Our simulations represent a reduced analysis, not a full mission analysis. Instead, it is geared towards investigating the contribution of the range and Doppler data types to the estimation of various parameters and comparing the results to those obtained from Sect. 4.1.

For both data types, we use a daily tracking pass of 2-hour duration, generating one independent measurement every 60 $\mathrm{s}$. The laser ranging data is weighted at $1 \mathrm{~cm}$, and the Doppler data at $0.01 \mathrm{~mm} / \mathrm{s}$ (see Sect. 6.1 for more detailed discussion). We do not simulate data for a solar separation angle smaller than $5^{\circ}$. For our simulations, we use the Tudat software. ${ }^{4}$

\section{Results}

Using the methods outlined in Sect. 4, we give the results of our analytical and numerical analyses.

\subsection{Analytical results}

Here, we present the results of the analysis method described in Sect. 4.1. For current radiometric systems, we use accuracies of $1 \mathrm{~m}$ in range and $0.04 \mathrm{~mm} / \mathrm{s}$ in range rate for $\Delta t_{i}=60 \mathrm{~s}$. For upcoming state-of-the-art systems, we assume $0.2-\mathrm{m}$ accuracy in radiometric range, and both 0.01 $\mathrm{mm} / \mathrm{s}$ at $60-\mathrm{s}$ integration time and $0.002 \mathrm{~mm} / \mathrm{s}$ at $1000-\mathrm{s}$ integration time (Sect. 2.1). For ILR, we assume an upper and lower bound on data quality of 2 and $10 \mathrm{~mm}$, respectively, a broad range which we derive from our discussion in Sect. 3.4. Evaluating Eqs. (11) and (12) to approximate the behaviour of the partial derivatives gives the results for the $\mathrm{SNR}_{h, q}$ from Eq. (9), shown in Fig. 2.

\footnotetext{
${ }^{4}$ Documentation at http://tudat.tudelft.nl; Code at http://github.com/ tudat/tudatBundle.
} 
Table 1 Estimated parameters for the numerical simulation cases

\begin{tabular}{lllllll}
\hline Simulation case & $\mathbf{x}_{i}\left(t_{j}\right)$ & $\mathbf{x}_{M}\left(t_{0}\right)$ & $\mathbf{x}_{L}$ & $\omega_{M}, \alpha_{M}, \delta_{M}$ & $(C, S)_{l, m}, k_{20} . . k_{22}$ & $\beta, J_{2} \odot$ \\
\hline Orbiters & $\checkmark$ & $\checkmark$ & & $\checkmark$ & $\checkmark$ & \\
Lander & & $\checkmark$ & $\checkmark$ & $\checkmark$ & & $\checkmark$ \\
\hline
\end{tabular}

-Radio Range-rate (case A), $\sigma=0.002 \mathrm{~mm} / \mathrm{s}, \Delta \mathrm{t}=1000 \mathrm{~s}$

- Radio Range-rate (case B), $\sigma=0.01 \mathrm{~mm} / \mathrm{s}, \Delta \mathrm{t}=60 \mathrm{~s}$

- Radio Range, $\sigma=0.2 \mathrm{~m}$

-Radio Range-rate, $\sigma=0.04 \mathrm{~mm} / \mathrm{s}, \Delta \mathrm{t}=60 \mathrm{~s}$

- Radio Range, $\sigma=1 \mathrm{~m}$

- Laser Range, $\sigma=0.002 \mathrm{~m}$

- Laser Range, $\sigma=0.01 \mathrm{~m}$

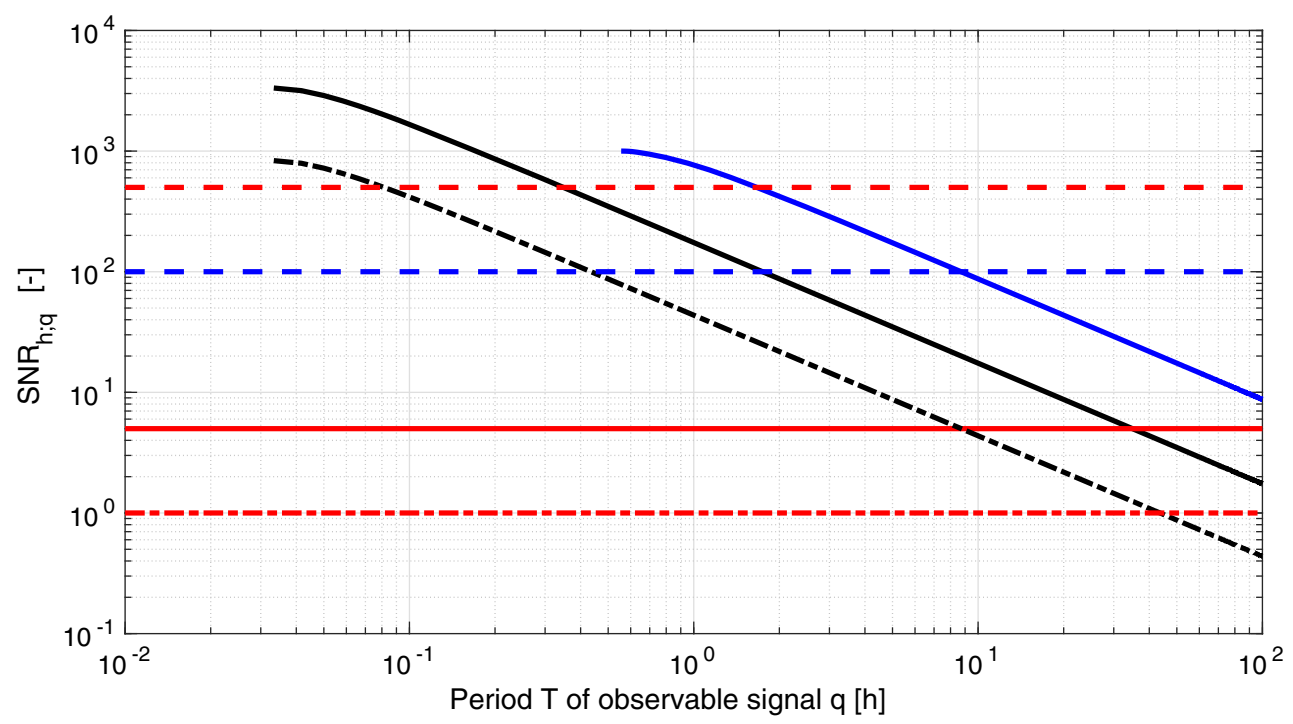

Fig. 2 Comparison of range and range-rate observable to purely periodic signals of amplitude $\omega$. Dashed line represents ILR, thick lines nextgeneration radiometric, dashed-dotted current radiometric. Adapted from Dirkx (2015)

The figure shows that the dual-frequency Doppler data at $\Delta t_{i}=60 \mathrm{~s}(\sigma=0.01 \mathrm{~mm} / \mathrm{s})$ and the ILR curves cross in the area of $0.33-1.65 \mathrm{~h}$ (for range data precision of 2-10 $\mathrm{mm}$ ), and therefore $\Xi_{q}<1$ for lower $T$ and $\Xi_{q}>1$ for larger $T$. This time interval is a particularly interesting one, as it contains the orbital period of many spacecraft orbiting rocky/icy bodies. For the $\Delta t_{i}=1000 \mathrm{~s}$ Doppler data, this time interval is shifted by a factor of 5 (as the 1000-s data have a precision 5 times better than the 60 -s data). For cases where $T<\Delta t_{i}$, however, a $2 \pi$ ambiguity arises in the estimation, requiring the use of a smaller $\Delta t_{i}$. Doppler data for orbiters typically have an integration time of $60 \mathrm{~s}$ or smaller. Reducing the integration time of the data does result in greater data volume, reducing the formal estimation errors if the noise is not correlated in time.

\subsection{Numerical results}

In this section, we show the results of the covariance analyses described in Sect. 4.2. These results are shown in Figs. $3,4,5$ and 6 . For these results, we use the formal error ratio $\epsilon_{q, s} / \epsilon_{q, \dot{s}}$ as figure of merit, instead of $\Xi_{q}$ from Eq. (14). For-

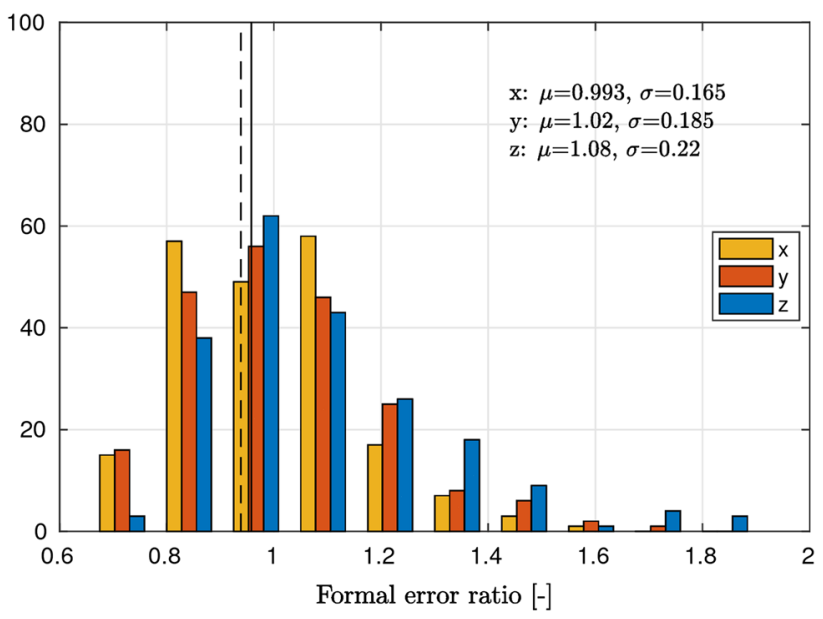

Fig. 3 Histograms of formal error ratio $\epsilon_{s} / \epsilon_{\dot{s}}$ for all arcs of the orbiter position component estimation, generated using the settings described in Sect. 4.2. Vertical black lines represent the analytical ratios $\Xi_{q}$ at the spacecraft orbital periods

mal errors are a more robust indicator of the relative strength of the observables, as it includes the difference in correlations 


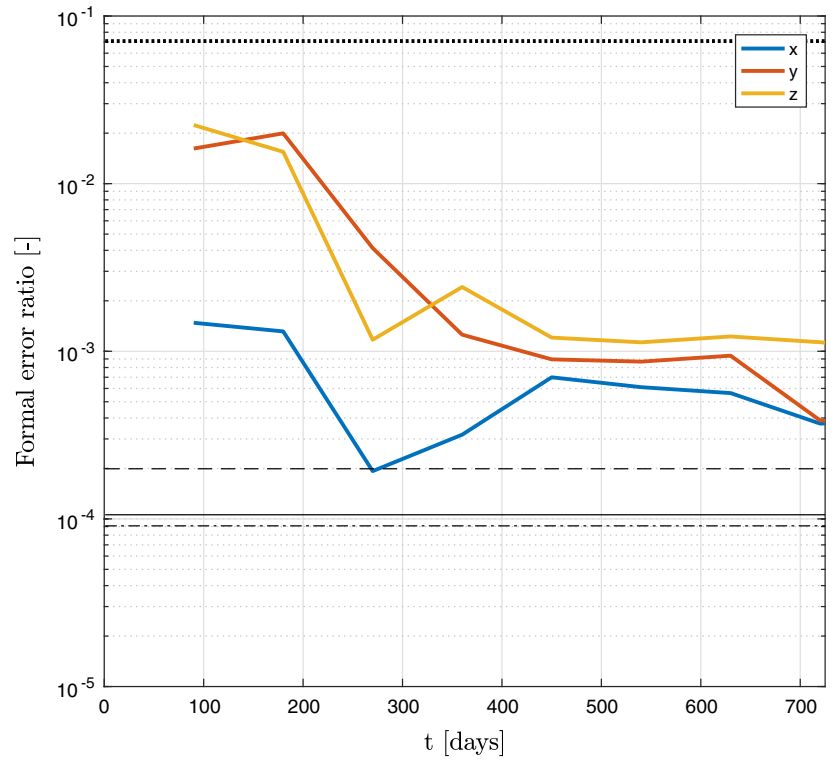

(a)

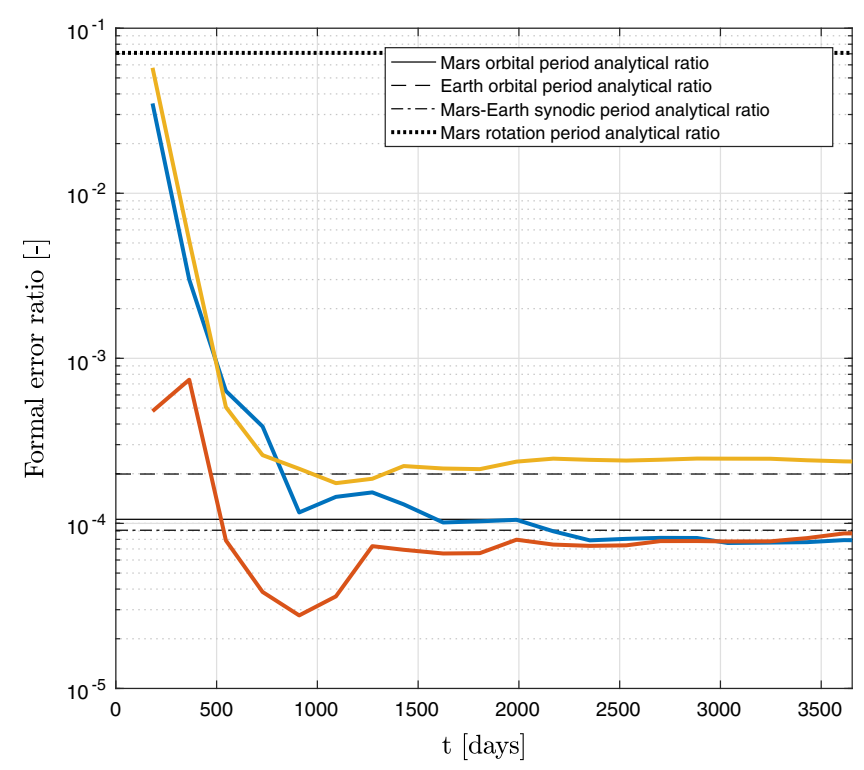

(b)

Fig. 4 Formal error ratios $\epsilon_{s} / \epsilon_{\dot{s}}$ for the Mars initial position components as a function of mission duration, from the settings described in Sect. 4.2. Horizontal black lines in a) represent analytical ratios $\Xi_{q}$ at relevant periods a orbiter simulation and $\mathbf{b}$ lander simulation

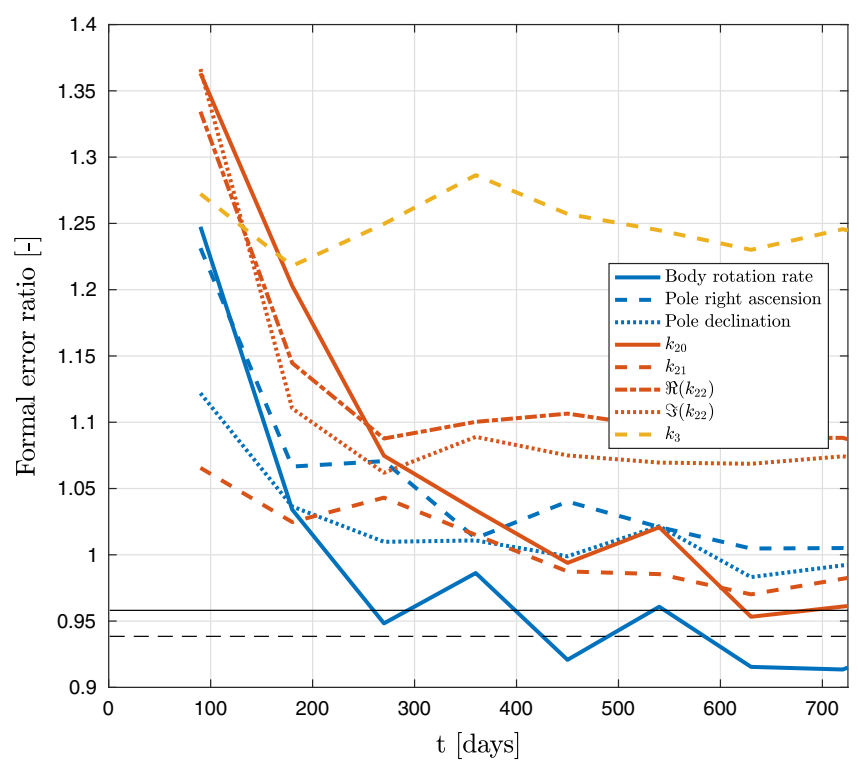

(a)

Fig. 5 Formal error ratios $\epsilon_{s} / \epsilon_{\dot{s}}$ for estimated parameters as a function of mission duration, from the settings described in Sect. 4.2. Horizontal black lines in a represent the analytical ratios $\Xi_{q}$ at the spacecraft

when performing Doppler-only and range-only estimation. In the analytical analysis of Sect. 5.1, formal errors cannot be obtained, as no estimation is performed. In the remainder of this section, we analyse how well the two figures of merit compare to one another. The analytical approach is a reasonable approximation if $\Xi_{q} \approx \epsilon_{q, s} / \epsilon_{q, \dot{s}}$.

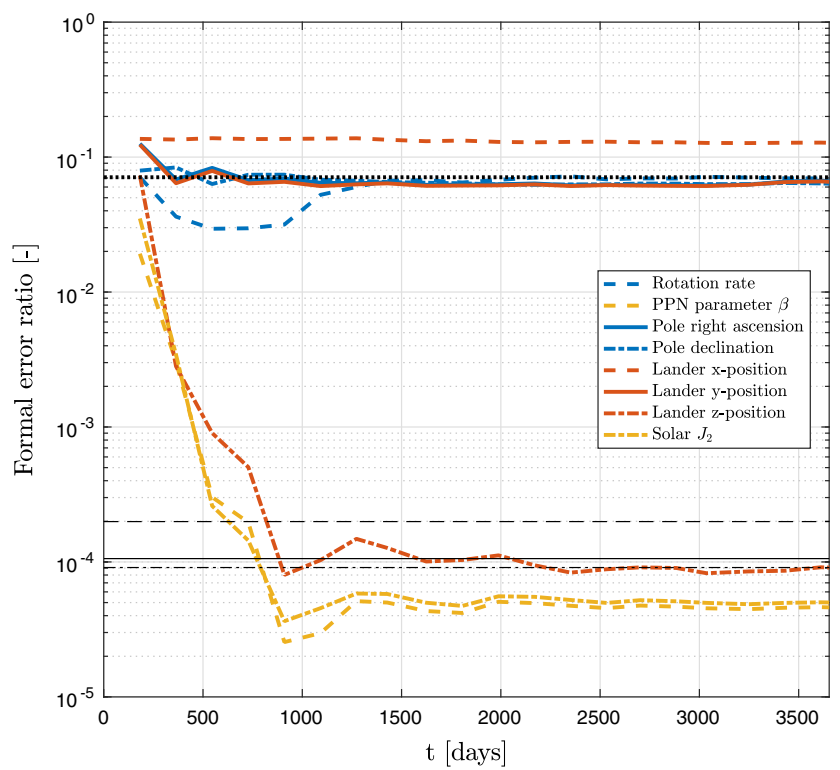

(b)

orbital periods. Horizontal black lines in $\mathbf{b}$ represent analytical ratios $\Xi_{q}$ at relevant periods, as in Fig. 4. a Orbiter simulation and $\mathbf{b}$ lander simulation

The orbiter arc initial position error ratios $\epsilon_{r_{i, 0}, s} / \epsilon_{r_{i, 0}, \dot{s}}$ are shown in Fig. 3. The mean ratio $(\approx 1.0)$ is slightly larger than the theoretical ratio $\Xi_{q}(\approx 0.95$, see Fig. 2 , with settings from Sect. 4.2). Deviations from the analytical value are primarily due to the correlations between the various param- 


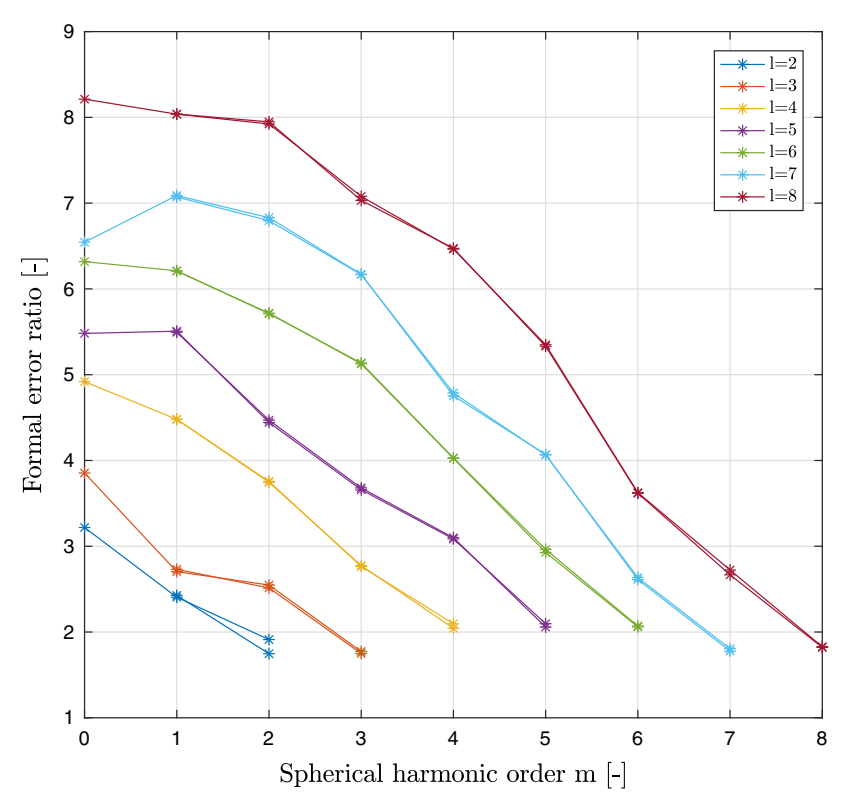

Fig. 6 Formal error ratios $\epsilon_{s} / \epsilon_{\dot{s}}$ for gravity field coefficients at $t=2$ years from the settings described in Sect. 4.2

eters, which are slightly worse for the range-only case than for the Doppler-only case.

The ratio $\epsilon_{r_{M}, s} / \epsilon_{r_{M}, \dot{s}}$ (Mars initial state) for the orbiter and lander simulations is shown in Fig. 4a, b, respectively. The theoretical ratios for several relevant periods (as taken from Fig. 2) are also indicated. For the lander simulations, the theoretical ratio is close to the value expected from a periodic signal at the Mars and Earth orbital frequencies. For the orbiter simulations, however, the ratios are a factor 5 higher than these analytical values (Fig. 4a). The correlations between $\mathbf{x}_{M}\left(t_{0}\right)$ and the other parameters are low for both data types. Since the analytical value for $\epsilon_{r_{M}, s} / \epsilon_{r_{M}, \dot{s}}$ is much more closely attained in the lander estimation (Fig. 4b), the discrepancy in the case of orbits indicates that the onceper-orbit signature of the spacecraft continues to have a significant effect on the estimation of Mars' initial state when obtained through the use of Eq. (15).

Rotational properties and Love numbers of Mars as estimated from orbiter data both have formal error ratios of around 1.0 (Fig. 5a). This is comparable to the orbiter initialstate estimation ratios (see Fig. 3) and indicates that the primary signature of these parameters is derived from a onceper-orbit signature. The range data perform slightly worse than the analytical result would indicate, due to stronger correlations between the parameters.

The error ratio for the gravity field coefficients $C_{l, m}$ and $S_{l, m}$ is shown in Fig. 6. These results show a clear trend in their formal error ratios, with $C_{l, l}$ and $S_{l, l}$ having a ratio about twice that of the initial state $\mathbf{r}_{0}$, increasing to roughly $(l+1)$ times that of the initial state for $C_{l, 0}$. Under the assumptions of the model outlined in Sect. 4.1, this would indicate that these parameters induce a periodic signature with a frequency of twice (for $m=l$ ) to $(l+1)$ times (for $m=0$ ) the orbital frequency.

Since the orbits we have used are close to polar, the time behaviour of the perturbation due to a given gravity field coefficient is mostly determined by $\nabla P_{l, m}(\sin \phi)$, with $P_{l, m}$ associated Legendre polynomials at degree $l$ and order $m$, and $\phi$ the body-fixed latitude of the spacecraft (e.g. Montenbruck and Gill 2000). A spherical harmonic coefficient at this degree and order causes an acceleration on the spacecraft that is linearly proportional to $P_{l+1, m+1}, P_{l+1, m}$ and $P_{l+1, m-1}$. For $P_{l, m}$, the number of zero crossings over a full orbit is 2 for $l=m$, while it is $l$ for $m=0$ and $m=1$.

Our results in Fig. 6 show that the influence of a function $P_{l, m}$ may be simplified to a sine function with the same number of zero crossings, for the purposes of our analytical comparison. This allows the analytical criterion in Fig. 2 to be used for approximating the relative contribution of range and Doppler data to gravity field estimation.

The error ratios of the parameters estimated in the lander scenario are shown in Fig. 5b. They clearly fall into two categories: those for which the formal error ratio is close to that predicted for signatures at Mars' rotational period (dotted black line; $\epsilon_{q, s} / \epsilon_{q, \dot{s}} \approx 0.07$ ) and those for Mars' orbital period (full black line; $\epsilon_{q, s} / \epsilon_{q, \dot{s}} \approx 10^{-4}$ ). All of Mars' rotational properties fall into the former category, as expected. The parameters impacting Mars' orbit ( $\beta$ and $J_{2} \odot$ ) fall into the latter category, but their formal error ratio is about a factor two smaller than expected from our analytical approximation (assuming the signal to be at Mars' orbital period). This difference is due to the correlation with the Mars initial velocity parameters, which is $<0.2$ for both $\beta$ and $J_{2} \odot$ in the range-only simulations and $0.6-0.7$ for the Doppleronly simulations. Finally, one of the components of the lander position is estimated poorly using Doppler data only, an effect well known and discussed by, for example, Le Maistre et al. (2013).

\section{Discussion}

Having shown the results of our analytical and numerical analyses in Sect. 5, we now discuss the potential application of ILR in planetary missions.

\subsection{Observation uncertainty comparison}

Section 5.1 gives the results of a conceptual criterion for comparing range and Doppler data using an analytical formulation. This is shown in Sect. 5.2 to approximate the results of the numerical covariance analysis reasonably well. However, both the analytical method and the covariance analysis suffer from common limitations: they ignore any differences 
in the measurement uncertainty probability distributions, and dynamical model errors are neglected.

Doppler tracking is close to being bias-free, and in the case of dual-frequency tracking, it has a noise spectrum that is close to Gaussian (Sect. 2.1). For laser tracking, the singleshot uncertainty distribution will be defined by the Gaussian pulse profile, convoluted with the detector impulse response in the case of single-photon detection (Sect. 3.1). Both the pulse profile and the detector response can be characterized to high accuracy (Sect. 3.2). However, as is the case in SLR/LLR, biases and instabilities at the several $\mathrm{mm}$ level will likely continue to be an issue (Sect. 3.4).

The issue of stability will be especially significant for lander tracking, where model uncertainties are expected to be the dominant source of estimation error (Sect. 3.4). For orbiter tracking, the dynamical model error will be more significant (compared to landers) and the impact of the precise observation noise spectrum less pronounced.

Dirkx et al. (2014b) analysed the impact of unresolved constant ILR biases for the Phobos Laser Ranging (PLR; Turyshev et al. 2010) mission. They used 5-mm Gaussian measurement noise and 5-mm constant unresolved bias, and obtained results indicate that the biases contribute about an order of magnitude more uncertainty to the estimated parameters than the Gaussian noise. For biases that vary quasi-randomly from pass to pass, this impact will in part average out. However, it will still cause the range-only simulations presented here to be more optimistic than the Doppler-only simulations, especially for the lander case. As such, any results from the criterion in Eq. (14) that indicate similar signatures on Doppler and range data should be interpreted as indicating Doppler data will likely continue to be the better choice of data type. Nevertheless, reducing system and observation biases in laser ranging systems is a continuous and ongoing priority in ILRS activities.

\subsection{Gravity fields and orbit determination}

Radio range measurements are poorly suited for estimating planetary gravity fields. The Doppler data are used as the primary input data type for current missions (e.g. Marty et al. 2009; Konopliv et al. 2011; Mazarico et al. 2014). The results in Sect. 5.1 indicate that the use of ILR becomes competitive for signatures with a period of $0.33-1.65 \mathrm{~h}$ for range data precisions of 2-10 $\mathrm{mm}$ (corresponding to range data accuracy at approximately sub-mm to several $\mathrm{mm}$ level, see Sect. 6.1). The analytical analysis is shown in Sect. 5.2 to provide a good approximation to the full numerical simulations for gravity field estimation (see Fig. 6). This indicates that Doppler data are the superior choice for gravity field estimation, with the possible exception of very low gravity field degrees. For low degrees (2-4), ILR could meaningfully complement the Doppler data, which would require exceptional stability of the range data. Since Doppler data are essentially bias-free, the competitive estimation of low-degree gravity fields will only be achievable by means of ILR if it is similarly (close to) bias-free (Sect. 6.1).

The comparatively poor sensitivity of ILR data to gravity field coefficients shows that a laser-only tracking system is unlikely to be a suitable design choice for a planetary orbiter. Any uncertainty in the target body gravity field will propagate into an increased error in the orbit determination of the spacecraft (e.g. Mazarico et al. 2012). Even if ILR is used on an orbiter for other applications, it should always be in tandem with a Doppler tracking system, which can be used to measure the high-frequency variations in the spacecraft dynamics, chiefly the influence of the target body's gravity field. One possible exception is in the case of lunar missions. The gravity field of the Moon has been determined to such extreme accuracy (Lemoine et al. 2014) that the impact of its uncertainty on a typical spacecraft's orbit determination will likely be negligible.

\subsection{Rotational and tidal characteristics}

For tidal parameters, the signature on orbiter dynamics will show a combination of the spacecraft's orbital period, the rotation rate of the body being orbited and the period of the tidal forcing. The results in Fig. 5b show that it mainly is the once-per-spacecraft-orbit signature that is dominant in determining the formal error ratio $\epsilon_{s} / \epsilon_{\dot{s}}$.

Similarly, rotational variations such as librations can have a broad range of periods, but significant variations typically do not have a period that is much smaller than the rotational period (e.g., Konopliv et al. 2006; Petit et al. 2010). As with tidal parameters, our results in Fig. 5a indicate that it is again the once-per-spacecraft-orbit signature that is dominant in determining $\epsilon_{s} / \epsilon_{\dot{s}}$. For most tidal and rotational parameters, the analytical approximation $(\approx 0.95)$ slightly overestimates the strength of the range data, as Fig. 5a shows formal error ratios $\approx 0.9-1.25$ (note that a higher ratio indicates a relatively weaker contribution of the range data).

This indicates that orbiter ILR data could be used to estimate tidal and rotational characteristics at a level that is comparable to that from Doppler data. Doing so will require a low level of unresolved systematic error in the range data, at the several mm level, assuming that (non-conservative) force modelling on the spacecraft does not limit the estimation quality. Moreover, as discussed in Sect. 6.2, the extraction of signals from the dynamics of orbiters will require the inclusion of a Doppler system, to prevent the uncertainty in short-periodic perturbations from degrading the quality of the estimation results. As a result, ILR could be used to supplement Doppler tracking in the determination of rotational and tidal characteristics from orbiter dynamics, but would not be the optimal choice as a dedicated system. 
Estimation of rotational properties from lander data is shown in Fig. 5b. Our analytical model closely predicts the $\epsilon_{s} / \epsilon_{\dot{s}}$ ratio, at the once-per-Martian-day frequency. Compared to orbiters, lander missions are more favourable for ILR, as the rotation period of a body (e.g. its day) is typically longer than the orbital period of a spacecraft.

Considering the typical rotational periods of bodies in our solar system, we can confidently state that laser range measurements to landers will be better suited for the estimation of rotational parameters than Doppler measurements will be. Figure 5b shows a factor 20 improvement of Mars rotational parameters from ILR compared to Doppler data. For some fast-rotating bodies such as Phobos, this factor would be reduced to $<10$. For bodies with much slower rotational periods, such as Ganymede and Mercury, the formal error from ILR could be $>100$ and $>1000$ times smaller than from Doppler data. However, at these levels of observational accuracy, many models will need to be improved to make full use of the data quality that would be available (Dirkx et al. 2014b).

Nevertheless, our results clearly show the exceptional strength that ILR can have in characterizing rotational motion and tidal deformation in the solar system, especially for landers. Similarly, it will be well suited for the determination of the $h_{2}$ and $l_{2}$ Love numbers [and possibly $k_{2}$, due to its influence on rotational dynamics (e.g. Williams et al. 2001)].

\subsection{Solar system ephemerides}

Figure 4 indicates that ILR is preferred over Doppler data for the determination of planetary ephemerides, which is unsurprising considering the current role of radio range data (Sect. 1). The relative contribution of range and Doppler data to the estimation of ephemerides is well approximated by the criterion in Eq. (14) for the case of lander missions. For orbiter data, Eq. (14) overestimates the relative contribution of the range data by a factor of up to 5. Clearly, the Doppler data continues to contribute to the determination of planetary ephemerides, by accurately extracting the orbiter dynamics from the observations. As a consequence, ephemeris determination will benefit from the combination of Doppler and ILR data, for reasons discussed in Sect. 6.2. Figure 5b shows that the parameters estimated jointly with ephemerides (here only $\beta$ and $J_{2, \odot}$ ) will benefit greatly from the use of ILR. These parameters are crucial in relativistic experiments (Will 2014). For the case of landers, their uncertainty is reasonably approximated by the analytical formulation. However, properly decorrelating these parameters using ILR data may require laser data to multiple targets.

Due to the scarcity of ILR data when it will be first implemented, there will be an imbalance of several orders of magnitude between the measurements used for creating solar system ephemerides. For instance, when using
ILR data from an Earth-Mars link, there will be mm-level range measurements for Earth and Mars, m-level (radiometric) range measurements for other solar system bodies at which orbiter/lander tracking data are available and km-level range measurements (radar) to bodies where no data from spacecraft tracking techniques are available. For many small bodies, no range data will be available at all, and ephemeris generation must be performed from astrometric data alone. This effect, as well as dynamical model error (Sect. 3.3), will degrade the fidelity of the orbit estimation of the bodies between which an ILR link is set up. Quantifying the exact requirements for a mission profile, tracking schedule, estimation settings, etc. for optimally exploiting ILR data in ephemeris generation will require a dedicated study.

\section{Conclusion-the science case for ILR}

The purpose of the article has been twofold. Firstly, we have given a detailed overview of the sources of uncertainty in both the realization and analysis of ILR data (Sect. 3). Secondly, we have compared the performance of ILR and radio Doppler data both analytically and numerically, to clarify the science case for ILR (Sects. 4-6).

Mm-precision normal points are feasible for ILR. Sub-cm accuracy of the data will be attainable, but reaching the mmlevel accuracy is hindered by both measurement instabilities and model uncertainties, similar to SLR/LLR (Sect. 3.4).

We have derived an analytical approximation of the sensitivity of ILR and radio Doppler data types, which is shown in Fig. 2, under the assumption of sinusoidal signatures on the data. This figure indicates that the signatures with a period of $0.33-1.65 \mathrm{~h}$ can be observed in the ILR and radio Doppler data at a similar signal-to-noise level. The results of our numerical covariance analysis largely validate the analytical approach, allowing Fig. 2 to be used as a conceptual design tool.

However, instabilities in the range data accuracy, which are not directly included in the simulations, will limit the performance of ILR data to the upper bounds of the 0.33 1.65 hour range. That is, ILR will start to be competitive for determining signatures of $>1.5-2 \mathrm{~h}$. For effects with a longer period than several hours, ILR data unambiguously provide a more accurate estimation, while Doppler data will continue to be the optimal data type for short-periodic effects.

ILR's weak sensitivity to short-periodic effects makes it a poor choice for gravity field estimation, with the possible exception of low-degree coefficients. Any orbiter with typical orbit determination requirements will continue to require Doppler data (with the possible exception of lunar missions). For typical mission profiles, ILR and Doppler data have a similar sensitivity to once-per-orbit effects (period of 1.5-2 h). Therefore, ILR will be valuable in complementing the 
Doppler data for the estimation of tidal and rotational characteristics from orbiter tracking. As expected, ILR is exceptionally well suited to generating ephemerides, although our analytical approximation overestimates its strength by a factor 5 for the case of orbiter tracking.

In addition, when used for lander tracking, ILR will be an excellent method for the estimation of both tidal and rotational characteristics of the target body. For a Mars lander, ILR data produce estimates that are about a factor 10 more accurate than the estimation from Doppler data. The strength of ILR improves even more for bodies with slower rotation rates.

We have focused on the estimation quality of orbits and geodetic parameters, concluding that the science case for landers is excellent, and it could serve a complementary role for orbiters. Owing to the highly accurate data that ILR will deliver, improvements in the various models entering not only the analysis, but also the interpretation of the estimation results, must be brought to a level where all data can be used to their full potential. Not only will this require significant theoretical effort, but it implies that more accurate knowledge must be obtained of various quantities that cannot be obtained from tracking data alone. Examples of synergistic data are magnetic field, heat flow, geological and seismic measurements, which will be important for the full characterization of a body's interior structure and composition. By combining these data from next-generation space missions with ILR, the full set of these measurements can be exploited to their full potential, allowing the study of planetary interiors to be brought to the next level.

Acknowledgements The authors are indebted to Luciano Iess for discussions on the strengths and weaknesses of planetary range and Doppler data, and to three anonymous reviewers, whose comments improved the clarity, conciseness and completeness of the manuscript. Part of this work was performed in the FP7 ESPaCE project, financially supported by the EC FP7 Grant Agreement 263466.

Open Access This article is distributed under the terms of the Creative Commons Attribution 4.0 International License (http://creativecomm ons.org/licenses/by/4.0/), which permits unrestricted use, distribution, and reproduction in any medium, provided you give appropriate credit to the original author(s) and the source, provide a link to the Creative Commons license, and indicate if changes were made.

\section{Appendix A: Semi-analytical approach-eccentric orbits}

A key exception for which the assumptions of Sect. 4.1 will not hold is when a spacecraft orbits with a substantial eccentricity, e.g. Juno ( $e \approx 0.95$ w.r.t. Jupiter $)$, Messenger $(e \approx 0.7$ w.r.t. Mercury), Mars Express ( $e \approx 0.57$ w.r.t. Mars). To extend the method of Sect. 4.1 to non-spherical orbits, we continue to use the criterion of Eq. (9), but compute the
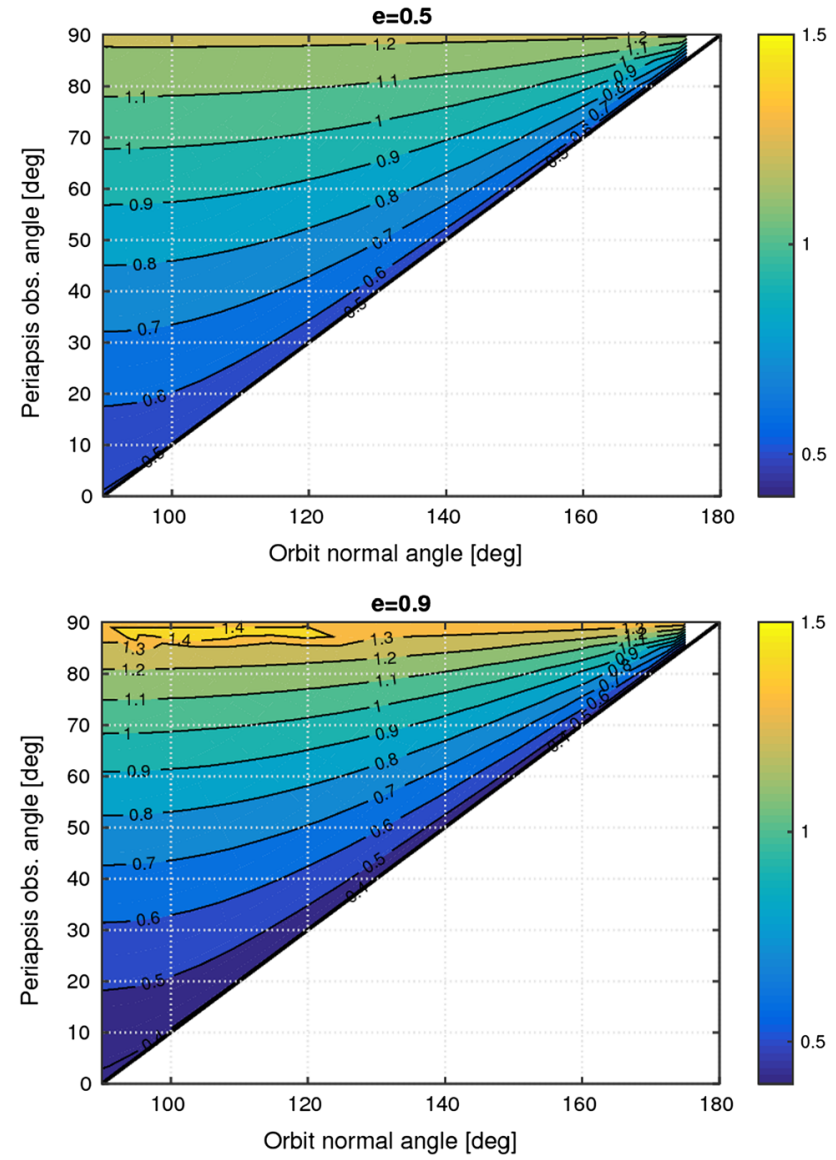

Fig. 7 Ratio of figures of merit $\Xi_{r_{0}}(e) / \Xi_{r_{0}}(e=0)$ for elliptical orbits and circular orbits as a function of viewing geometry. The colour scale denotes this ratio. Note that the periapsis distance $r_{\mathrm{p}}$ is set equal in the $e=0$ and $e \neq 0$ simulations

partial derivatives from the orbits directly using the method of (Moyer 2000), instead of imposing them to behave sinusoidally. We limit ourselves to taking $q$ as the initial position $\mathbf{r}_{0}$ of the spacecraft w.r.t. the planet it is orbiting.

We vary the eccentricity from 0 to 1 and assess the influence on the behaviour of $\Xi_{q}$. For an eccentric orbit, the influence of the geometry of the orbital plane w.r.t. the observation line of sight needs to be analysed. We parameterize this geometric dependency by two angles: the angle between the line-of-sight vector and the spacecraft orbital plane, and the angle between the line-of-sight vector and the spacecraft orbital velocity vector at periapsis.

Using the methodology outlined above, we have generated values of $\Xi_{q}$ (with $q$ the initial position $\mathbf{r}_{0}$, and computing the associated $\Xi_{r_{0}}$ as the root sum square of the constituent vector components of $\partial h / \partial \mathbf{q}$ ) for elliptical Kepler orbits under a full range of observational geometries w.r.t. the observer line of sight.

As a test case, we use a Mars orbiter for our analysis. When generating the values of $\Xi_{r_{0}}$ for two cases, one with $e=0$ 
and one with $e>0$, we find that using the same spacecraft semi-major axis for both does not lead to insightful results. Instead, when using the same spacecraft periapsis distance $r_{\mathrm{p}}=a(1-e)$ for the two cases, the results for zero and nonzero eccentricities can be related much more intuitively. We show the results of this analysis in Fig. 7, where we plot the ratios of $\Xi_{r_{0}}(e) / \Xi_{r_{0}}(e=0)$, using the same $r_{\mathrm{p}}$ to compute the two values of $\Xi_{r_{0}}$.

From this figure, it can be seen that even for substantial eccentricities, the ratio $\Xi_{r_{0}}(e) / \Xi_{r_{0}}(e=0)$ remains close to 1 for a broad range of eccentricities and observational geometries. In our simulations $(e \leq 0.9)$, the results deviate from the analytical ratio by less than $50 \%$ in all cases and less than $10 \%$ in most cases. This indicates that the analytical criterion shown in Fig. 2 continues to be largely applicable even for large eccentricities. This result greatly simplifies the first-order analysis of the added value of an ILR system, as it allows the analytical results for spherical orbits to be used for arbitrary eccentricities.

When using Fig. 2 to analyse the comparative strength of range and Doppler data for elliptical orbits with period $\omega_{\mathrm{P}}$, the following scaling should be applied to obtain the value of $\omega$ at which Fig. 2 is to be read:

$\omega=(1-e)^{-1.5} \omega_{\mathrm{P}}$

so that for elliptical orbits the Doppler data continue to be the preferred method over ILR for a larger range of values of $\omega_{\mathrm{P}}$.

\section{References}

Abshire J, Sun X, Neumann G, McGarry J, Zagwodzki T, Jester P, Riris H, Zuber M, Smith D (2006) Laser pulses from Earth detected at Mars. In: Conference on lasers and electro-optics, pp 1-2

Altamimi Z, Collilieux X, Métivier L (2011) ITRF2008: an improved solution of the International Terrestrial Reference Frame. J Geod 85:457-473

Asmar SW, Armstrong JW, Iess L, Tortora P (2005) Spacecraft Doppler tracking: noise budget and accuracy achievable in precision radio science observations. Radio Sci 40:2001

Bauer S (2017) Application of one-way laser ranging data to the Lunar Reconnaissance Orbiter (LRO) for time transfer, clock characterization and orbit determination. $\mathrm{PhD}$ thesis, Technische Universität, Berlin

Bauer S, Oberst J, Hussmann H, Dirkx D, Mao D, Neumann G, Mazarico E, Torrence M, McGarry J, Smith D, Zuber M (2016) Demonstration of orbit determination for the Lunar Reconnaissance Orbiter using one-way laser ranging data. Planet Space Sci 129:32-46

Bauer S, Hussmann H, Oberst J, Dirkx D, Mao D, Neumann GA, Mazarico E, Torrence MH, McGarry JF, Smith DE, Zuber MT (2017) Analysis of one-way laser ranging data to LRO, time transfer and clock characterization. Icarus 283:38-54

Bertone S, Poncin-Lafitte CL, Rosenblatt P, Lainey V, Marty J-C, Angonin M-C (2018) Impact analysis of the transponder time delay on radioscience observables. Adv Space Res 61(1):89-96
Bertotti B, Iess L, Tortora P (2003) A test of general relativity using radio links with the Cassini spacecraft. Nature 425:374-376

Birnbaum KM, Chen Y, Hemmati H (2010) Precision optical ranging by paired one-way time of flight. In: SPIE conference series, vol 7587

Blazej J, Prochazka I, Kodet J, Linhart P (2014) Indoor demonstration of free-space picosecond two-way time transfer on single photon level. In: Laser communication and propagation through the atmosphere and oceans III, vol 9224 of SPIE Conference Series

Bocanegra-Bahamón T, Calvés GM, Gurvits L, Duev D, Pogrebenko S, Cimò G, Dirkx D, Rosenblatt P (2018) Planetary radio interferometry and Doppler experiment (PRIDE) technique: a test case of the mars express phobos flyby-II. Doppler tracking: formulation of observed and computed values, and noise budget. Astron Astrophys 609:A59

Chandler J, Pearlman M, Reasenberg R, Degnan J (2005) Solar-system dynamics and tests of general relativity with planetary laser ranging. In: Proceedings of the 14th international workshop on laser ranging

Chen Y, Birnbaum KM, Hemmati H (2013) Active laser ranging over planetary distances with millimeter accuracy. Appl Phys Lett 102(24): 241107

Christophe B, Andersen PH, Anderson JD, Asmar S, Bério P, Bertolami O, Bingham R, Bondu F, Bouyer P, Bremer S, Courty J-M, Dittus H, Foulon B, Gil P, Johann U, Jordan JF, Kent B, Lämmerzahl C, Lévy A, Métris G, Olsen O, Pàramos J, Prestage JD, Progrebenko SV, Rasel E, Rathke A, Reynaud S, Rievers B, Samain E, Sumner TJ, Theil S, Touboul P, Turyshev S, Vrancken P, Wolf P, Yu N (2009) Odyssey: a solar system mission. Exp Astron 23:529-547

Degnan J (1996) Compact laser ranging transponders for interplanetary laser and time transfer. In: Proceedings of 10th international workshop on laser ranging, pp 24-31

Degnan J (2017) Challenges to achieving millimeter accuracy normal points in conventional multiphoton and $\mathrm{kHz}$ single photon SLR systems. In: Proceedings of the 2017 ILRS Technical workshop

Degnan J (2002) Asynchronous laser transponders for precise interplanetary ranging and time transfer. J Geodyn 34:551-594

Degnan J J (2008) Laser transponders for high-accuracy interplanetary laser ranging and time transfer. In: Dittus $\mathrm{H}$, Lammerzahl C, Turyshev S G (eds) Lasers, clocks and drag-free control: exploration of relativistic gravity in space, vol 349. Astrophysics and space science library. Springer, New York, p 231

Dehant V, Park R, Dirkx D, Iess L, Neumann G, Turyshev S, Van Hoolst $T$ (2017) Survey of capabilities and applications of accurate clocks: directions for planetary science. Space Sci Rev 212(3-4):14331451

Dirkx D (2015) Interplanetary laser ranging-analysis for implementation in planetary science mission. $\mathrm{PhD}$ thesis, Delft University of Technology

Dirkx D, Noomen R, Prochazka I, Bauer S, Vermeersen L (2014a) Influence of atmospheric turbulence on planetary transceiver laser ranging. Adv Space Res 54(11):2349-2370

Dirkx D, Vermeersen L, Noomen R, Visser P (2014b) Phobos laser ranging: numerical geodesy experiments for Martian system science. Planet Space Sci 99:84-102

Dirkx D, Noomen R, Visser P, Bauer S, Vermeersen L (2015) Comparative analysis of one- and two-way planetary laser ranging concepts. Planet Space Sci 117:159-176

Dirkx D, Lainey V, Gurvits LI, Visser PNAM (2016a) Dynamical modelling of the Galilean moons for the JUICE mission. Planet Space Sci 134:82-95

Dirkx D, Noomen R, Visser P, Gurvits L, Vermeersen L (2016b) Spacetime dynamics estimation from space mission tracking data. Astron Astrophys 587:A156 
Dirkx D, Gurvits LI, Lainey V, Lari G, Milani A, Cimò G, BocanegraBahamon T, Visser P (2017) On the contribution of PRIDE-JUICE to Jovian system ephemerides. Planet Space Sci 147:14-27

Duev DA, Molera Calvés G, Pogrebenko SV, Gurvits LI, Cimó G, Bocanegra Bahamon T (2012) Spacecraft VLBI and Doppler tracking: algorithms and implementation. Astron Astrophys 541:A43

Duev DA, Pogrebenko SV, Cimò G, Calvés GM, Bahamón TB, Gurvits LI, Kettenis MM, Kania J, Tudose V, Rosenblatt P et al (2016) Planetary radio interferometry and Doppler experiment (PRIDE) technique: a test case of the Mars express phobos fly-by. Astron Astrophys 593:A34

Exertier P, Bonnefond P, Deleflie F, Barlier F, Kasser M, Biancale R, Menard Y (2006) Contribution of laser ranging to Earth's sciences. Comptes Rendus Geosci 338:958-967

Exertier P, Samain E, Martin N, Courde C, Laas-Bourez M, Foussard C, Guillemot P (2014) Time transfer by laser link: data analysis and validation to the ps level. Adv Space Res 54:2371-2385

Exertier P, Belli A, Lemoine J (2017) Time biases in laser ranging observations: a concerning issue of space geodesy. Adv Space Res 60(5):948-968

Fienga A, Laskar J, Morley T, Manche H, Kuchynka P, Le PoncinLafitte C, Budnik F, Gastineau M, Somenzi L (2009) INPOP08, a 4-D planetary ephemeris: from asteroid and time-scale computations to ESA Mars express and venus express contributions. Astron Astrophys 507:1675-1686

Fienga A, Laskar J, Kuchynka P, Manche H, Desvignes G, Gastineau M, Cognard I, Theureau G (2011) The INPOP10a planetary ephemeris and its applications in fundamental physics. Celest Mech Dyn Astron 111:363-385

Folkner W, Finger M (1990) Preliminary error budget for an optical ranging system: range, range rate and differenced range observables. Interplanet Netw Progress Rep 42(101):121-135

Folkner WM, Williams JG, Boggs DH, Park RS, Kuchynka P (2014) The planetary and lunar ephemerides DE430 and DE431. Interplanet Netw Progress Rep 42(196):C1

Gardner C (1976) Effects of random path fluctuations on the accuracy of laser ranging systems. Appl Opt 15:2539-2545

Hulley G, Pavlis E (2007) A ray-tracing technique for improving satellite laser ranging atmospheric delay corrections, including the effects of horizontal refractivity gradients. J Geophys Res (Solid Earth) 112(B11):B06417

Iess L, Jacobson RA, Ducci M, Stevenson DJ, Lunine JI, Armstrong JW, Asmar SW, Racioppa P, Rappaport NJ, Tortora P (2012) The tides of Titan. Science 337(6093):457-459

Iess L, Benedetto MD, James N, Mercolino M, Simone L, Tortora P (2014) Astra: Interdisciplinary study on enhancement of the end-to-end accuracy for spacecraft tracking techniques. Acta Astronautica 94(2):699-707

Iess L, Folkner W, Durante D, Parisi M, Kaspi Y, Galanti E, Guillot T, Hubbard W, Stevenson D, Anderson J et al (2018) Measurement of Jupiters asymmetric gravity field. Nature 555(7695):220

Iorio L (2013) LETSGO: A spacecraft-based mission to accurately measure the solar angular momentum with frame-dragging. Acta Astronautica 86:149-157

Jones DL, Folkner WM, Jacobson RA, Jacobs CS, Dhawan V, Romney J, Fomalont E (2015) Astrometry of Cassini With the VLBA to improve the saturn ephemeris. Astron J 149:28

Kirchner G, Koidl F (2014) SLR calibration issues-example: Graz. In: Proceedings of the 19th international workshop on laser ranging, number 3154

Klioner SA (2008) Relativistic scaling of astronomical quantities and the system of astronomical units. Astron Astrophys 478:951-958

Kliore A, Anderson J, Armstrong J, Asmar S, Hamilton C, Rappaport N, Wahlquist H, Ambrosini R, Flasar F, French R et al. (2004) Cassini radio science. In: The Cassini-Huygens Mission. Springer, Berlin, pp 1-70
Kodet J, Prochazka I (2012) Note: Optical trigger device with sub-picosecond timing jitter and stability. Rev Sci Instrum 83(3):036101

Konopliv AS, Yoder CF, Standish EM, Yuan D-N, Sjogren WL (2006) A global solution for the Mars static and seasonal gravity, Mars orientation, Phobos and Deimos masses, and Mars ephemeris. Icarus 182:23-50

Konopliv AS, Asmar SW, Folkner WM, Karatekin Ö, Nunes DC, Smrekar SE, Yoder CF, Zuber MT (2011) Mars high resolution gravity fields from MRO, Mars seasonal gravity, and other dynamical parameters. Icarus 211:401-428

Kral K, Prochazka I, Hamal K (2005) Optical signal path delay fluctuations caused by atmospheric turbulence. Opt Lett 30(14):17671769

Le Maistre S, Rosenblatt P, Rambaux N, Castillo-Rogez JC, Dehant V, Marty J-C (2013) Phobos interior from librations determination using Doppler and star tracker measurements. Planet Space Sci $85: 106-122$

Lemoine FG, Goossens S, Sabaka TJ, Nicholas JB, Mazarico E, Rowlands DD, Loomis BD, Chinn DS, Neumann GA, Smith DE, Zuber MT (2014) GRGM900C: a degree 900 lunar gravity model from GRAIL primary and extended mission data. Geophys Res Lett 41(10):3382-3389

Luo Y-J, Xia Y, Li G-Y (2009) Scientific value of the laser ranging of Asteroid Icarus. Chin Astron Astrophys 33:440-449

Mao D, McGarry JF, Mazarico E, Neumann GA, Sun X, Torrence MH, Zagwodzki TW, Rowlands DD, Hoffman ED, Horvath JE, Golder JE, Barker MK, Smith DE, Zuber MT (2017) The laser ranging experiment of the Lunar Reconnaissance Orbiter: Five years of operations and data analysis. Icarus 283:55-69

Marty JC, Balmino G, Duron J, Rosenblatt P, Le Maistre S, Rivoldini A, Dehant V, van Hoolst T (2009) Martian gravity field model and its time variations from MGS and Odyssey data. Planet Space Sci 57:350-363

Mazarico E, Rowlands DD, Neumann GA, Smith DE, Torrence MH, Lemoine FG, Zuber MT (2012) Orbit determination of the lunar reconnaissance orbiter. J Geod 86:193-207

Mazarico E, Genova A, Goossens S, Lemoine FG, Neumann GA, Zuber MT, Smith DE, Solomon SC (2014) The gravity field, orientation, and ephemeris of Mercury from MESSENGER observations after three years in orbit. J Geophys Res Planets 119(12):2417-2436

Merkowitz S, Dabney P, Livas J, McGarry J, Neumann G, Zagwodzki T (2007) Laser ranging for gravitational, lunar and planetary science. Int J Modern Phys D 16:2151-2164

Milani A, Gronchi G (2010) Theory of orbit determination. Cambridge University Press, Cambridge

Molera Calvés G, Pogrebenko S, Cimò G, Duev D, BocanegraBahamón T, Wagner J, Kallunki J, De Vicente P, Kronschnabl G, Haas R et al (2014) Observations and analysis of phase scintillation of spacecraft signal on the interplanetary plasma. Astron Astrophys 564:A4

Montenbruck O, Gill E (2000) Satellite orbits: models, methods, and applications. Physics and astronomy online library. Springer, Berlin

Moyer T (2000) Formulation for observed and computed values of deep space network data types for navigation, vol 2. JPL deep-space communications and navigation series. Wiley, New York

Murphy T (2001) Statistics of photon arrival time. Tech. Rep. http://tmurphy.physics.ucsd.edu/apollo/doc/arrival.pdf. Accessed 13 July 2018

Murphy TW (2013) Lunar laser ranging: the millimeter challenge. Rep Progress Phys 76(7):076901

Neumann GA, Cavanaugh JF, Coyle DB, McGarry J, Smith DE, Sun X, Torrence M, Zagwodski TW, Zuber MT (2008) Laser ranging at interplanetary distances. In: Proceedings of the 16th international workshop on laser ranging 
Noda H, Kunimori H, Mizuno T, Senshu H, Ogawa N, Takeuchi H, Moore C, Pollard A, Yamaguchi T, Namiki N et al (2017) Laser link experiment with the Hayabusa 2 laser altimeter for in-flight alignment measurement. Earth Planets Space 69(1):2

Oberst J, Lainey V, Le Poncin-Lafitte C, Dehant V, Rosenblatt P, Ulamec S, Biele J, Spurmann J, Kahle R, Klein V, Schreiber U, Schlicht A, Rambaux N, Laurent P, Noyelles B, Foulon B, Zakharov A, Gurvits L, Uchaev D, Murchie S, Reed C, Turyshev SG, Gil J, Graziano M, Willner K, Wickhusen K, Pasewaldt A, Wählisch M, Hoffmann H (2012) GETEMME-a mission to explore the Martian satellites and the fundamentals of solar system physics. Exp Astron 34:243-271

Otsubo T, Appleby GM (2003) System-dependent center-of-mass correction for spherical geodetic satellites. J Geophys Res (Solid Earth) 108:2201

Panek P, Prochazka I, Kodet J (2010) Time measurement device with four femtosecond stability. Metrologia 47:L13-L16

Pearlman MR, Degnan JJ, Bosworth JM (2002) The international laser ranging service. Adv Space Res 30:135-143

Petit G, Luzum B et al (2010) IERS conventions (2010). IERS Technical Note, No, p 36

Prochazka I, Blazej J, Kodet J (2011) Measurement of the optical to electrical detection delay in the detector for ground-to-space laser time transfer. Metrologia 48:L13-L16

Prochazka I, Kodet J, Blazej J, Kirchner G, Koidl F, Wang P (2017a) Identification and calibration of one-way delays in satellite laser ranging systems. Adv Space Res 59(10):2466-2472

Prochazka I, Kodet J, Eckl J, Blazej J (2017b) Note: Large active area solid state photon counter with 20 ps timing resolution and $60 \mathrm{fs}$ detection delay stability. Rev Sci Instrum 88(10):106105

Prochazka I, Blazej J, Kodet J (2012) New technologies for time transfer with picoseconds precision and accuracy. In: 2012 IEEE international frequency control symposium (FCS), p 1-7

Prochazka I, Kodet J, Panek P, Schreiber U (2011) Novel concept of sub-picosecond timing system and its applications in fundamental metrology. In: Frequency Control and the European Frequency and Time Forum, pp 1-4

Reasenberg RD, Shapiro II, MacNeil PE, Goldstein RB, Breidenthal JC, Brenkle JP, Cain DL, Kaufman TM, Komarek TA, Zygielbaum AI (1979) Viking relativity experiment-verification of signal retardation by solar gravity. Astrophys J 234:L219-L221

Rivoldini A, van Hoolst T, Verhoeven O, Mocquet A, Dehant V (2011) Geodesy constraints on the interior structure and composition of Mars. Icarus 213:451-472

Rothacher M, Angermann D, Artz T, Bosch W, Drewes H, Gerstl M, Kelm R, König D, König R, Meisel B, Müller H, Nothnagel A, Panafidina N, Richter B, Rudenko S, Schwegmann W, Seitz M, Steigenberger P, Tesmer S, Tesmer V, Thaller D (2011) GGOS$\mathrm{D}$ : homogeneous reprocessing and rigorous combination of space geodetic observations. J Geod 85:679-705
Samain E, Exertier P, Courde C, Fridelance P, Guillemot P, Laas-Bourez M, Torre J-M (2015) Time transfer by laser link: a complete analysis of the uncertainty budget. Metrologia 52:423

Schreiber KU, Hiener M, Holzapfel B, Michaelis H, Brandl N, Haufe K-H, Lauber P, Neidhardt A (2009) Altimetry and transponder ground simulation experiment. Planet Space Sci 57:1485-1490

Smith D, Zuber M, Sun X, Neumann G, Cavanaugh J, McGarry J, Zagwodzki T (2006) Two-way laser link over interplanetary distance. Science 311:53-53

Soffel M, Klioner SA, Petit G, Wolf P, Kopeikin SM, Bretagnon P, Brumberg VA, Capitaine N, Damour T, Fukushima T, Guinot B, Huang T-Y, Lindegren L, Ma C, Nordtvedt K, Ries JC, Seidelmann PK, Vokrouhlický D, Will CM, Xu C (2003) The IAU 2000 resolutions for astrometry, celestial mechanics, and metrology in the relativistic framework: explanatory supplement. Astron J 126:2687-2706

Sośnica K, Thaller D, Dach R, Jäggi A, Beutler G (2013) Impact of loading displacements on SLR-derived parameters and on the consistency between GNSS and SLR results. J Geod 87:751-769

Stevens ML, Parenti RR, Willis MM, Greco JA, Khatri FI, Robinson BS, Boroson DM (2016) The lunar laser communication demonstration time-of-flight measurement system: overview, on-orbit performance, and ranging analysis. In: Free-space laser communication and atmospheric propagation XXVIII, vol 9739 of SPIE conference series, p 973908

Teyssandier P, Le Poncin-Lafitte C (2008) General post-Minkowskian expansion of time transfer functions. Class Quantum Gravity 25(14): 145020

Thornton C, Border J (2000) Radiometric tracking techniques for deepspace navigation, vol 1. JPL deep-space communications and navigation series. Wiley, New York

Turyshev SG, Shao M, Nordtvedt KL (2004) Experimental design for the lator mission. Int J Modern Phys D 13:2035-2063

Turyshev SG, Farr W, Folkner WM, Girerd AR, Hemmati H, Murphy TW, Williams JG, Degnan JJ (2010) Advancing tests of relativistic gravity via laser ranging to Phobos. Exp Astron 28:209-249

Will C (2014) The confrontation between general relativity and experiment. Living Rev Relativ 17:4

Williams JG, Boggs DH, Yoder CF, Ratcliff JT, Dickey JO (2001) Lunar rotational dissipation in solid body and molten core. J Geophys Res 106:27933-27968

Zuber MT, Smith DE, Zellar RS, Neumann GA, Sun X, Katz RB, Kleyner I, Matuszeski A, McGarry JF, Ott MN, Ramos-Izquierdo LA, Rowlands DD, Torrence MH, Zagwodzki TW (2010) The lunar reconnaissance orbiter laser ranging investigation. Space Sci Rev 150:63-80 